\title{
Unbounded Stress in Subregular Phonology
}

\author{
Yiding Hao \\ Yale University \\ New Haven, CT, USA \\ yiding.hao@yale.edu
}

\author{
Samuel Andersson \\ Yale University \\ New Haven, CT, USA \\ samuel .andersson@yale.edu
}

\begin{abstract}
This paper situates culminative unbounded stress systems within the subregular hierarchy for functions. While Baek (2018) has argued that such systems can be uniformly understood as input tier-based strictly local constraints, we show here that default-to-oppositeside and default-to-same-side stress systems belong to distinct subregular classes when they are viewed as functions that assign primary stress to underlying forms. While the former system can be captured by input tier-based input strictly local functions, a subsequential function class that we define here, the latter system is not subsequential, though it is weakly deterministic according to McCollum et al.'s (2018) non-interaction criterion. Our results motivate the extension of recently proposed subregular language classes to subregular functions and argue in favor of McCollum et al.'s definition of weak determinism over that of Heinz and Lai (2013).
\end{abstract}

\section{Introduction}

The treatment of unbounded stress (Baek, 2018), Uyghur backness harmony (Mayer and Major, 2018), and Sanskrit n-retroflexion (Graf and Mayer, 2018) in subregular phonology has given rise to a rich collection of extensions of the tierbased strictly local languages (TSL; Heinz et al., 2011) as formal descriptions of the typology of phonotactic dependencies. These language classes formalize the notion of local dependencies defined on tiers. While the TSL languages assume that each segment is either projected to the tier or not, the extensions allow for rich tier-projection schemata that are sensitive to local context.

Meanwhile, the formal study of phonological processes has shown that mappings from underlying representations to surface representations often exhibit a form of locality analogous to the notion captured by TSL languages. This insight is formalized by the input strictly local (ISL; Chandlee, 2014), output strictly local (OSL; Chandlee et al., 2015), and input-output strictly local (IOSL; Chandlee et al., In prep) functions, proposed as functional counterparts of the TSL languages. Chandlee (2014) argues that most phonological processes are captured by these classes of functions, and of the ones that are not, Heinz and Lai (2013) and McCollum et al. (2018) propose two versions of the weakly deterministic functions that describe non-deterministic harmony patterns.

This paper examines culminative unbounded stress systems as string-to-string mappings. Baek (2018) analyzes these systems as phonotactic constraints and shows that they are not TSL in general. To capture them, Baek defines the tierbased strictly local languages with structural features (TSL-SF), an extension of TSL in which the tier-projection mechanism is sensitive to the position of segments within prosodic units. The TSLSF languages were later subsumed by Graf and Mayer's (2018) input-output tier-based strictly local (IO-TSL) languages, in which the tierprojection mechanism is implemented by an arbitrary IOSL function. As mappings, we show that default-to-opposite-side (DO) stress systems can be captured using a similar approach. Examples of such systems include stressing the leftmost long vowel and assigning rightmost stress in the absence of long vowels. We propose the input tier-based input strictly local (I-TISL) functions as a functional analogue of the generalized tierprojection mechanism of the IO-TSL languages. Based on the stress system of Abkhaz, we advocate for a tier projection that is slightly more general than the restricted mechanism used in Baek. Next, we show that default-to-same-side (DS) stress systems, such as that of Lhasa Tibetan, are not subsequential. Examples of DS systems include those in which the leftmost long vowel is stressed, and 
the leftmost vowel is stressed when all vowels are short. We argue that DS systems can be naturally captured using McCollum et al.'s (2018) definition of weak determinism but not using Heinz and Lai's (2013) definition, therefore arguing in favor of the former definition.

This paper is structured as follows. Section 2 states basic notation and definitions used throughout this paper. Section 3 defines the I-TISL functions and shows that they can capture the DO stress system of Abkhaz. Section 4 considers Lhasa Tibetan and its relation to the two definitions of weak determinism. Section 5 presents a discussion of these results and their connection with analyses of stress based on metrical grid theory. Section 6 concludes.

\section{Preliminaries}

As usual, $\mathbb{N}$ denotes the set of nonnegative integers. $\Sigma$ and $\Gamma$ denote finite alphabets not including the left and right word boundary symbols $\rtimes$ and $\ltimes$, respectively. The length of a string $x$ is denoted by $|x|$, and $\lambda$ denotes the empty string. Alphabet symbols are identified with strings of length 1 , and individual strings are identified with singleton sets of strings. For $k \in \mathbb{N}, \alpha^{k}$ denotes $\alpha$ concatenated with itself $k$-many times, $\alpha^{*}$ denotes $\bigcup_{i=0}^{\infty} \alpha^{i}$, and $\alpha^{+}$denotes $\alpha \alpha^{*}$. The longest common prefix of a set of strings $A$ is the longest string $\operatorname{lcp}(A)$ such that every string in $A$ begins with $\operatorname{lcp}(A)$. The $k$ suffix of a string $x$, denoted $\operatorname{suff}^{k}(x)$, is the string consisting of the last $k$-many symbols of $\rtimes^{k} x$. The reverse of a string $x=x_{1} x_{2} \ldots x_{n}$, denoted $x^{\mathrm{R}}$, is the string $x_{n} x_{n-1} \ldots x_{1}$. For any functions $f: A \rightarrow B$ and $g: B \rightarrow C$, the notation $g \circ f$ represents the function given by $(g \circ f)(x)=g(f(x))$. A function $f: \Sigma^{*} \rightarrow \Gamma^{*}$ is same-length if for all $x \in \Sigma^{*},|f(x)|=|x|$.

\subsection{Subsequential Functions}

This subsection presents an algebraic definition of the subsequential functions, analogous to the Nerode-Myhill characterization of the regular languages. We use the translations of a function $f$ to describe the possible behaviors of a subsequential finite-state transducer (SFST) for $f$, and we identify each translation of $f$ with a state of the minimal SFST for $f$.

Definition 1. Let $f: \Sigma^{*} \rightarrow \Gamma^{*}$. We define the function $f^{\leftarrow}: \Sigma^{*} \rightarrow \Gamma^{*}$ by

$$
f^{\leftarrow}(x):=\operatorname{lcp}\left(\left\{f(x y) \mid y \in \Sigma^{*}\right\}\right) .
$$

For any $x, y \in \Sigma^{*}, f_{x}(y)$ denotes the string such that $f(x y)=f^{\leftarrow}(x) f_{x}(y)$. We refer to the function $f_{x}^{\rightarrow}$ as the translation of $f$ by $x$ and to $f^{\leftarrow}$ as $f$ top. ${ }^{1}$

Intuitively, $f^{\leftarrow}(x)$ refers to the output of the minimal SFST for $f$ after reading the input $x$, and the translation $f_{x} \rightarrow$ describes the behavior of the minimal SFST upon reading further input symbols.

Definition 2 (Raney, 1958). A function $f: \Sigma^{*} \rightarrow$ $\Gamma^{*}$ is subsequential if the set $\left\{f_{x} \mid x \in \Sigma^{*}\right\}$ is finite. We say that $f$ is left-subsequential if it is subsequential and right-subsequential if the function $g: \Sigma^{*} \rightarrow \Gamma^{*}$ defined by $g(x):=f\left(x^{\mathrm{R}}\right)^{\mathrm{R}}$ is subsequential. We say that $f$ is sequential or left-sequential if $f$ is subsequential and $f=f^{\leftarrow}$. We say that $f$ is right-sequential if the function $g: \Sigma^{*} \rightarrow \Gamma^{*}$ defined by $g(x):=f\left(x^{\mathrm{R}}\right)^{\mathrm{R}}$ is sequential.

The strictly local functions are defined by assuming that each translation corresponds to an $i$ suffix of the input and a $j$-suffix of the output.

Definition 3 (Chandlee et al., In prep). For $i, j \in$ $\mathbb{N}$, a function $f: \Sigma^{*} \rightarrow \Gamma^{*}$ is $i, j$-input-output strictly local ( $i, j$-IOSL) if for every $x, y \in \Sigma^{*}$, if $\operatorname{suff}^{i-1}(x)=\operatorname{suff}^{i-1}(y)$ and $\operatorname{suff}^{j-1}\left(f^{\leftarrow}(x)\right)=$ suff $^{j-1}\left(f^{\leftarrow}(y)\right)$, then $f_{x}^{\rightarrow}=f_{y}$. If the function $g(x):=f\left(x^{\mathrm{R}}\right)^{\mathrm{R}}$ is $i, j$-IOSL, then $f$ is right $i, j$-input-output strictly local (right $i, j$-IOSL). A function is $i$-input strictly local ( $i$-ISL) if it is $i, 1$ IOSL and $j$-output strictly local ( $j$-OSL) if it is $1, j$-IOSL. A function is input-output strictly local (IOSL), input strictly local (ISL), or output strictly local (OSL) if if is $i, j$-IOSL, $i$-ISL, or $j$-OSL for some $i, j \in \mathbb{N}$, respectively. A function is homomorphic if it is sequential and 1,1-IOSL.

Since there are only finitely many possible $i$ suffixes and $j$-suffixes, it is clear that all IOSL functions are subsequential.

\section{DO Stress and Tier Projection}

In culminative unbounded stress systems, primary stress is assigned to either the first or last syllable that fulfills a particular criterion-e.g., having a long vowel. In the absence of such syllables, primary stress is assigned to either the first or last syllable by default. DO stress systems are those

${ }^{1}$ This terminology follows Sakarovitch (2009, pp. 692693). In the transducer inference literature, Oncina et al. (1993) refer to $f_{x} \rightarrow$ as the tails of $x$ in $f$, and Chandlee et al. (2015) refer to $f^{\leftarrow}$ as the prefix function associated to $f$. 
in which qualifying syllables closest to one word edge receive stress, while the syllable closest to the other edge receives stress by default. In DS stress systems, qualifying syllables closest to one word edge receive stress, and the syllable closest to the same edge receives stress by default. For example, in the typology of Hayes (1995), leftmost heavy otherwise rightmost (LHOR) and rightmost heavy otherwise leftmost systems (RHOL) are DO, while leftmost heavy otherwise leftmost (LHOL) and rightmost heavy otherwise rightmost (RHOR) systems are DS.

This section considers the DO stress system of Abkhaz, which we describe in Subsection 3.1. Subsection 3.2 defines the I-TSL functions and shows how they can capture the Abkhaz stress system.

\subsection{Abkhaz Stress}

Below we illustrate the stress system of the standard Abzhuy variety of Abkhaz ([abk], Northwest Caucasian), as analyzed by Dybo (1977; refined by Spruit, 1986 and Trigo, 1992). The Abkhaz stress system depends on a set of phonologicallycontrastive accentual specifications. In the data considered here, every syllable of every morpheme is lexically specified as either dominant (D) or recessive (R; see Spruit, 1986 for accentual specifications which do not align with syllable boundaries). The so-called Dybo's Rule for stress states: assign primary stress to the leftmost D not immediately followed by another D (Spruit, 1986, p. 38). We mark dominant syllables by underlining, and hyphens indicate morpheme boundaries. Evidence for accentual specifications can be found in Spruit (1986).

The Abkhaz stress pattern is illustrated in Tables $1-3$. When a word contains only a single dominant syllable, it receives the primary stress. When there is a span of multiple dominant syllables which are all adjacent, the rightmost such syllable is stressed. When there are multiple spans of adjacent $\mathrm{D}$ syllables, the rightmost $\mathrm{D}$ of the leftmost span is stressed. In words with only D syllables, as well as in words with only $\mathrm{R}$ syllables, stress is final.

\subsection{I-TISL Functions}

Let us now define the I-TISL functions and show how they can handle the Abkhaz stress system. To do so, we extend the notion of tier projection used in the TSL languages (Heinz et al., 2011; Baek,

\begin{tabular}{|c|c|}
\hline Form & Translation \\
\hline a- $\widehat{t}^{\mathrm{h}} \mathrm{a}-\mathrm{ga}$ & '(the) hoe' \\
\hline 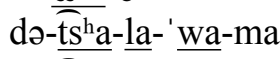 & 'does (s)he usually go?' \\
\hline 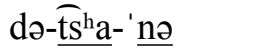 & '(s)he having gone' \\
\hline
\end{tabular}

Table 1: The rightmost consecutive dominant syllable receives primary stress (Spruit, 1986, pp. 50, 53).

\begin{tabular}{|c|c|}
\hline Form & Translation \\
\hline 'a-va-ț'a-ra & 'to put next to' \\
\hline də-' gəla-g ${ }^{\mathrm{w}} \mathrm{u} \int \mathrm{a}-\mathrm{ma}$ & $\begin{array}{l}\text { 'did (s)he go and stand, } \\
\text { alas?' }\end{array}$ \\
\hline a-' ' $^{\mathrm{w}} \mathrm{ak}{ }^{\prime}{ }^{\mathrm{j}} \mathrm{am} \boldsymbol{\text { sa }}$ & '(the) poniard' \\
\hline
\end{tabular}

Table 2: Only the leftmost span of Ds contains a primary stress (Spruit, 1986, pp. 44, 47, 73-74).

2018; Mayer and Major, 2018; Graf and Mayer, 2018) to the case of subregular functions. There, tier projections are formalized as functions that delete certain symbols of their inputs.

Definition 4. A tier-projection function on $\Sigma$ is a function $\pi: \Sigma^{*} \rightarrow \Sigma^{*}$ such that for any $x=$ $x_{1} x_{2} \ldots x_{n} \in \Sigma^{*}$ we have $\pi(x)=y_{1} y_{2} \ldots y_{n}$, where for each $i$, either $y_{i}=x_{i}$ or $y_{i}=\lambda$.

TSL languages make use of tier projection by only enforcing local dependencies based on symbols projected to the tier, thereby bypassing symbols not projected to the tier. To apply the tier projection system to strictly local functions, we only consider symbols on the tier when enforcing strict locality. Whereas $i$-ISL functions require that their translations by a string $x$ correspond to the last $(i-1)$-many symbols of $x$, tier-based $i$-ISL functions associate translations with the last $(i-1)$ many symbols on the tier, which we identify with $\operatorname{suff}^{i-1}\left(\pi^{\leftarrow}(x)\right)$.

Definition 5. A function $f: \Sigma^{*} \rightarrow \Gamma^{*}$ is $i$ input tier-based $j$-input strictly local ( $i$-I- $j$-TISL) if there exists an $i$-ISL tier projection function $\pi$ on $\Sigma$ such that for all $x, y \in \Sigma^{*}$, if $\operatorname{suff}^{j-1}\left(\pi^{\leftarrow}(x)\right)=$ $\operatorname{suff}^{j-1}\left(\pi^{\leftarrow}(y)\right)$, then $f_{x}^{\rightarrow}=f_{y}$. We call $\pi$ a tier projection for $f$. A function is input tier-based input strictly local (I-TISL) if it is $i$-I- $j$-TISL for some $i$ and $j$.

We formalize the Abkhaz stress system as follows. Alphabet symbols represent individual syllables; dominant and recessive syllables are represented as D and R, respectively. Stressed syllables are represented as $\mathrm{D}$ and R. Following the 


\begin{tabular}{ll}
\hline Form & Translation \\
\hline$\underline{\mathrm{a}-\mathrm{p}^{\mathrm{h}} \mathrm{a}-\mathrm{\prime}}$ ' $\underline{\text { ra }}$ & 'to pleat' \\
maa-'k'ə & 'one handle" \\
\hline
\end{tabular}

Table 3: Stress is final when all syllables have the same accentual status (Spruit, 1986, pp. 45-46).

discussion from Subsection 3.1, the Abkhaz stress function replaces the leftmost $\mathrm{D}$ not followed by another D with D. If the input does not contain any Ds, then the final $R$ is replaced with $R$.

Definition 6. The Abkhaz stress function $\alpha$ : $\{D, R\}^{*} \rightarrow\{D, R, D, R\}^{*}$ is defined as follows. For $i \geq 0, j>0$, and $y \in\{\lambda\} \cup \mathrm{R}\{\mathrm{D}, \mathrm{R}\}^{*}$,

$$
\begin{aligned}
\alpha\left(\mathrm{R}^{i} \mathrm{D}^{j} y\right) & :=\mathrm{R}^{i} \mathrm{D}^{j-1} \mathbf{D} y \\
\alpha\left(\mathrm{R}^{j}\right) & :=\mathrm{R}^{j-1} \hat{\mathrm{R}} \\
\alpha(\lambda) & :=\lambda .
\end{aligned}
$$

Proposition 7. The Abkhaz stress function is 2-I3-TISL.

Proof. Let $\pi:\{\mathrm{D}, \mathrm{R}\}^{*} \rightarrow\{\mathrm{D}, \mathrm{R}\}^{*}$ be the tierprojection function defined as follows.

- Any D not preceded by another D is projected.

- Any R preceded by a D is projected.

It is easy to see that $\pi$ is 2-ISL. Observe that for any $x, \pi(x) \in(\mathrm{DR})^{*}\{\lambda, \mathrm{D}\}$.

We now show that $\alpha$ is 2-I-3-TISL with tier projection $\pi$. To that end, we need to show that each translation $\alpha_{x}^{\rightarrow}$ is determined by $\operatorname{suff}^{2}\left(\pi^{\leftarrow}(x)\right)$. Observe that there are four possible values for $\operatorname{suff}^{2}\left(\pi^{\leftarrow}(x)\right): \rtimes \rtimes, \rtimes \mathrm{D}, \mathrm{DR}$, or RD. We consider each of these cases one-by-one.

- Suppose $\operatorname{suff}^{2}\left(\pi^{\leftarrow}(x)\right)=\rtimes \rtimes$. This means that $x=\mathrm{R}^{i}$ for some $i \geq 0$. Stress has not yet been assigned, so $\alpha^{\leftarrow}(x)=\mathrm{R}^{i-1}$ if $i>0$ and $\alpha^{\leftarrow}(x)=\lambda$ otherwise. For any $y \in\{D, R\}^{*}$,

$$
\alpha_{x}(y)= \begin{cases}\mathrm{R} \alpha(y), & i>0 \\ \alpha(y), & i=0 .\end{cases}
$$

- Suppose $\operatorname{suff}^{2}\left(\pi^{\leftarrow}(x)\right)=\rtimes \mathrm{D}$. This means that $x=\mathrm{R}^{i} \mathrm{D}^{j}$, where $j>0$ and $i \geq 0$. Stress has not yet been assigned, so $\alpha^{\leftarrow}(x)=$ $\mathrm{R}^{i} \mathrm{D}^{j-1}$. For any input of the form $\mathrm{D}^{m} \mathbf{R}^{n} y$, where $m, n \geq 0$ and $y \in\{\mathrm{D}, \mathrm{R}\}^{*}$,

$$
\alpha_{x}\left(\mathrm{D}^{m} \mathbf{R}^{n} y\right)= \begin{cases}\mathrm{D}^{m} \dot{\mathrm{D}}^{n} y, & n>0 \\ \mathrm{D}^{m} y \dot{\mathrm{D}}, & \mathrm{R}^{n} y \in \mathrm{D}^{*} .\end{cases}
$$

- Suppose $\operatorname{suff}^{2}\left(\pi^{\leftarrow}(x)\right) \in\{\mathrm{DR}, \mathrm{RD}\}$. Now, stress has already been assigned, so $\alpha^{\leftarrow}(x)=$ $\alpha(x)$ and for all $y, \alpha_{x}(y)=y$.

In all four cases, we have seen that $\alpha_{x}$ does not depend on $x$, though it does depend on $\operatorname{suff}^{2}\left(\pi^{\leftarrow}(x)\right)$. Therefore, $\alpha$ is 2-I-3-TISL.

In the construction described above, the fact that $\pi$ is allowed to be 2-ISL enables $\pi$ to only project symbols marking the boundaries between contiguous spans of Ds and Rs. In the original tier-projection mechanism of Heinz et al. (2011), for each symbol $u \in \Sigma$, either all tokens of $u$ must be projected to the tier, or no tokens of $u$ may be projected.

Definition 8. A function is tier-based $j$-input strictly local ( $j$-TISL) if it is $1-\mathrm{I}-j$-TISL and has a homomorphic tier projection. A function is tierbased input strictly local (TISL) if it is $j$-TISL for some $j$.

To justify the use of a 2-ISL tier projection, we show that the primitive tier-projection mechanism does not suffice to capture Abkhaz stress.

Proposition 9. The Abkhaz stress function is not TISL.

Proof. Let $\pi:\{\mathrm{D}, \mathrm{R}\}^{*} \rightarrow\{\mathrm{D}, \mathrm{R}\}^{*}$ be a homomorphic tier projection. We will show that for every $j>0$, there exist $x, y, z \in\{\mathrm{D}, \mathrm{R}\}^{*}$ such that $\operatorname{suff}^{j-1}\left(\pi^{\leftarrow}(x)\right)=\operatorname{suff}^{j-1}\left(\pi^{\leftarrow}(y)\right)$, but $\alpha_{x}(z) \neq$ $\alpha_{y}(z)$.

Fix $j>0$, and suppose $\pi$ projects D. Then, $\operatorname{suff}^{j-1}\left(\pi^{\leftarrow}\left(\mathrm{D}^{j}\right)\right)=\operatorname{suff}^{j-1}\left(\pi^{\leftarrow}\left(\mathrm{DRD}^{j}\right)\right)=$ $\mathrm{D}^{j-1}$, but $\alpha_{\mathrm{D}^{j}}(\mathrm{R})=\mathrm{DR}$, while $\alpha_{\mathrm{DRD}^{j}}(\mathrm{R})=$ R. Next, suppose $\pi$ projects $\mathrm{R}$. Then, $\operatorname{suff}^{j-1}\left(\pi^{\leftarrow}\left(\mathrm{R}^{j}\right)\right)=\operatorname{suff}^{j-1}\left(\pi^{\leftarrow}\left(\mathrm{DR}^{j}\right)\right)=\mathrm{R}^{j-1}$, but $\alpha_{\mathrm{R}^{j}}(\mathrm{D})=\mathrm{RD}$, while $\alpha_{\mathrm{DR}^{j}}^{\overrightarrow{(D)}}=\mathrm{D}$. Finally, suppose $\pi$ projects neither $\mathrm{R}$ nor $\mathrm{D}$. Then, $\operatorname{suff}^{j-1}\left(\pi^{\leftarrow}(\mathrm{R})\right)=\operatorname{suff}^{j-1}\left(\pi^{\leftarrow}(\mathrm{DR})\right)=\rtimes^{j-1}$, but $\alpha_{\mathrm{R}}(\mathrm{D})=\mathrm{RD}$, while $\alpha_{\mathrm{DR}}(\mathrm{D})=\mathrm{D}$. Therefore, $\alpha$ is not $j$-TISL for any $j$ and for any $\pi$.

In addition to exceeding the power of TISL functions, our tier projection is also considerably more sophisticated than the projection used in Baek (2018). There, Baek formalizes LHOL, LHOR, RHOL, and RHOR systems as phonotactic constraints and projects heavy, stressed, wordinitial, and word-final syllables to the tier. She achieves this by using the primitive tier-projection mechanism with an augmented alphabet in which 
syllables are marked as being word-initial, wordfinal, or word-medial. To avoid feature coding (Rogers, 1997), Baek stipulates that syllables cannot be marked in any other way. Since Dybo's Rule is sensitive to more nuanced structural information, we argue that arbitrary ISL tier projections are required for DO stress systems in general.

\section{DS Stress and Weak Determinism}

We have now shown that the DO stress system of Abkhaz is I-TISL. This section turns to DS stress systems. Subsection 4.1 introduces the DS stress system of Lhasa Tibetan, and in Subsection 4.2 we show that this stress system is not subsequential. Subsection 4.3 considers two definitions of the weakly deterministic functions, proposed by Heinz and Lai (2013) and McCollum et al. (2018), and argue that the latter definition more naturally describes the Lhasa Tibetan stress system than the former.

\subsection{Lhasa Tibetan Stress}

We describe here the stress system of Tibetan ([bod], Sino-Tibetan) using data from the Lhasa variety, as described by Dawson (1980). The descriptive generalization about Tibetan stress is as follows: primary stress falls on the leftmost long vowel, and if there are no long vowels, on the leftmost vowel. Stress is indicated with the IPA primary stress diacritic, and long vowels are underlined. Below we illustrate the generalizations about Tibetan stress. All of our data come from Gordon (2007, p. 37), who in turn cites Dawson (1980). The same data can also be found in Odden (1979), who cites personal communication with $\mathrm{N}$. Nornang.

The Lhasa Tibetan stress pattern is illustrated in Tables 4 and 5. When a word contains one or more long vowels, the leftmost long vowel receives the primary stress. When there are no long vowels, the leftmost vowel has primary stress. Thus, this is an example of a DS stress system.

\begin{tabular}{|c|c|}
\hline Form & Translation \\
\hline ám'tố: & 'person from Amdo' \\
\hline $\begin{array}{l}\text { khá' pá: } \\
\text { 'tý:tû́: }\end{array}$ & $\begin{array}{l}\text { 'telephone' } \\
\text { 'shirt' }\end{array}$ \\
\hline
\end{tabular}

Table 4: The leftmost long vowel receives primary stress.

\begin{tabular}{ll}
\hline Form & Translation \\
\hline 'láptá & 'school' \\
'núgú & 'pen' \\
'wòmá & 'milk' \\
\hline
\end{tabular}

Table 5: Default stress is initial.

\subsection{Non-Subsequentiality}

Intuitively, the behavior of an SFST is to scan its input from left to right, emitting output symbols deterministically as it does so. This paradigm of computation is problematic for DS stress systems such as that of Lhasa Tibetan. In order to determine whether or not the first syllable of its input should be stressed, an SFST implementing Lhasa Tibetan stress must scan the entire input to check for the presence of long vowels. However, once the SFST has determined that an input does not have any long vowels, it no longer has access to the initial syllable, and therefore cannot mark it as stressed. The following discussion makes this intuition rigorous by showing that the Lhasa Tibetan stress system is not subsequential.

We formalize the Lhasa Tibetan stress system as follows. Syllables with long-vowel nuclei are represented as $\mathrm{H}$, while syllables with short-vowel nuclei are represented as L. Stressed syllables are represented as $\mathrm{H}$ and Ĺ, respectively. If an input contains at least one $\mathrm{H}$, then the first $\mathrm{H}$ receives stress. Otherwise, the first syllable receives stress.

Definition 10. The Tibetan stress function $\tau$ : $\{\mathrm{H}, \mathrm{L}\}^{*} \rightarrow\{\mathrm{H}, \mathrm{L}, \dot{\mathrm{H}}, \mathbf{L}\}^{*}$ is defined as follows. For $i \geq 0, j>0$, and $y \in\{\mathrm{H}, \mathrm{L}\}^{*}$,

$$
\begin{aligned}
\tau\left(\mathrm{L}^{i} \mathrm{H} y\right) & :=\mathrm{L}^{i} \hat{\mathrm{H}} y \\
\tau\left(\mathrm{L}^{j}\right) & :=\mathrm{LL}^{j-1} \\
\tau(\lambda) & :=\lambda .
\end{aligned}
$$

Proposition 11. The Tibetan stress function is not subsequential.

Proof. We will show that $\tau$ has infinitely many translations. Consider a string of the form $\mathrm{L}^{i}$, where $i>0$. Observe that $\tau\left(\mathrm{L}^{i} \mathrm{H}\right)=\mathrm{L}^{i} \dot{\mathrm{H}}^{\text {and }}$ $\tau\left(\mathrm{L}^{i}\right)=\mathrm{LL}^{i-1}$. Therefore,

$$
\operatorname{lcp}\left(\left\{\tau\left(\mathrm{L}^{i} \mathrm{H}\right), \tau\left(\mathrm{L}^{i}\right)\right\}\right)=\lambda,
$$

so $\tau^{\leftarrow}\left(\mathrm{L}^{i}\right)=\lambda$, hence $\tau_{\mathrm{L}^{i}}(\mathrm{H})=\mathrm{L}^{i} \hat{\mathrm{H}}$. But this means that if $i \neq j$, then

$$
\mathrm{L}^{i} \hat{\mathrm{H}}=\tau_{\mathrm{L}^{i}}(\mathrm{H}) \neq \tau_{\mathrm{L}^{j}}(\mathrm{H})=\mathrm{L}^{j} \dot{\mathrm{H}} .
$$


Thus, each possible value of $i$ induces a distinct translation $\tau_{\mathrm{L}^{i}}$, so we conclude that $\tau$ is not subsequential.

\subsection{Weak Determinism}

The current subregular approach to nonsubsequential processes is represented by the weakly deterministic functions, a class proposed by Heinz and Lai (2013) in order to distinguish the unattested sour grapes harmony process from attested harmony processes. ${ }^{2}$ Dominant/recessive and stem-controlled vowel harmony (Heinz and Lai, 2013; McCollum et al., 2018), Tutrugbu ATR harmony (McCollum et al., 2018), and Copperbelt Bemba tone spreading (McCollum et al., 2018; Smith and O'Hara, 2019) have so far been shown to be non-subsequential but weakly deterministic in the sense of Heinz and Lai.

Definition 12 (Heinz and Lai, 2013). A function $f: \Sigma^{*} \rightarrow \Gamma^{*}$ is markup-free weakly deterministic if there exist functions $g: \Sigma^{*} \rightarrow \Sigma^{*}$ and $h: \Sigma^{*} \rightarrow$ $\Gamma^{*}$ such that

- $f=h \circ g$;

- either $g$ is left-subsequential and $h$ is rightsubsequential or $g$ is right-subsequential and $h$ is left-subsequential; and

- for all $x \in \Sigma^{*},|g(x)| \leq|x|$.

Elgot and Mezei (1965) show that every finitestate function can be decomposed into a leftsubsequential function and a right-subsequential function. In their construction, the first function in the composition encodes state information into its input, which allows the second function to determinize its computation. The above definition attempts to prohibit this kind of encoding by requiring that $g$ cannot introduce new alphabet symbols or increase the length of its input. McCollum et al. (2018) argue that a limited form of state encoding is still possible under Heinz and Lai's criterion, and instead advocate for a more explicit notion of noninteraction between the two functions.

Definition 13 (McCollum et al., 2018). Let $f$ : $\Sigma^{*} \rightarrow \Gamma^{*}$ be a same-length function. ${ }^{3}$ Fix $x \in \Sigma^{*}$, and write $x=x_{1} x_{2} \ldots x_{n}$ and $y=y_{1} y_{2} \ldots y_{n}$ so

\footnotetext{
${ }^{2}$ However, it is currently unknown whether this class of functions is distinct from the class of finite-state functions.

${ }^{3} \mathrm{McC}$ collum et al. additionally require that $f$ be sequential. We relax this assumption here.
}

that for each $i, x_{i} \in \Sigma$ and $y_{i} \in \Gamma$. The $\mu$-factors of $x$ with respect to $f$ are the set

$$
\mu(f, x):=\left\{\left\langle i, x_{i}, y_{i}\right\rangle \mid y_{i} \neq x_{i}\right\} .
$$

The basic intuition behind McCollum et al.'s criterion is that the two functions in the decomposition cannot feed or bleed one another. They take this to mean that neither function can cause the other to change its behavior for some position of the input.

Definition 14 (McCollum et al., 2018). A function $f: \Sigma^{*} \rightarrow \Gamma^{*}$ is interaction-free weakly deterministic if there exist an alphabet $\Delta \supseteq \Sigma$ and functions $g: \Sigma^{*} \rightarrow \Delta^{*}$ and $h: \Delta^{*} \rightarrow \Gamma^{*}$ such that

- $f=h \circ g$;

- either $g$ is left-subsequential and $h$ is rightsubsequential or $g$ is right-subsequential and $h$ is left-subsequential; and

- for all $x \in \Sigma^{*}, \mu(f, x)=\mu(g, x) \cup \mu(h, x) .{ }^{4}$

This criterion naturally describes the Tibetan stress function. We can decompose this function into a left-subsequential function that assigns stress to the leftmost $\mathrm{H}$ and a right-subsequential function that assigns initial stress in the absence of an $\mathrm{H}$. These two functions do not interact, since each only assigns stress if the other does not.

Proposition 15. The Tibetan stress function is interaction-free weakly deterministic.

Proof. Let us define $g:\{\mathrm{H}, \mathrm{L}\}^{*} \rightarrow\{\mathrm{H}, \mathrm{L}, \mathrm{H}\}^{*}$ and $h:\{\mathrm{H}, \mathrm{L}, \mathrm{H}\}^{*} \rightarrow\{\mathrm{H}, \mathrm{L}, \mathrm{H}, \mathrm{L}\}^{*}$ as follows. For $i \geq 0, y \in\{\mathrm{H}, \mathrm{L}\}^{*}$, and $z \notin \mathrm{L}^{+}$,

$$
\begin{aligned}
g\left(\mathrm{~L}^{i} \mathrm{H} y\right) & :=\mathrm{L}^{i} \mathrm{H} y \\
g\left(\mathrm{~L}^{i}\right) & :=\mathrm{L}^{i} \\
h(z) & :=z \\
h\left(\mathrm{LL}^{i}\right) & :=\mathbf{L L}^{i} .
\end{aligned}
$$

It is clear that $\tau=h \circ g, g$ is left-sequential, and $h$ is right-subsequential. Observe that $h(x)=x$ if $g(x) \neq x$ and $h(x) \neq x$ if $g(x)=x$, so for all $x$, either $\mu(h, x)=\varnothing$ and $\mu(\tau, x)=\mu(g, x)$ or $\mu(g, x)=\varnothing$ and $\mu(\tau, x)=\mu(h, x)$. Therefore, $\tau$ is interaction-free weakly deterministic.

\footnotetext{
${ }^{4}$ Note that this criterion implicitly requires that $f, g$, and $h$ all be same-length.
} 
On the other hand, it is difficult to see how the Tibetan stress function can be made to satisfy Heinz and Lai's definition of weak determinism. The decomposition presented above violates the markup-free criterion because $g$ introduces new alphabet symbols to its input. This is an inherent property of stress assignment, since inputs must be annotated with stress markers. In order to satisfy the markup-free criterion, then, any stress assigned by the first function must be encoded without using a designated stress marker. We conjecture that no such decomposition exists.

\section{Discussion}

We have now given two separate subregular treatments of DO and DS stress systems. Subsection 5.1 shows how our implementation of the Abkhaz and the Tibetan stress functions mirrors existing phonological analyses of stress systems according to metrical grid theory. Subsection 5.2 discusses the implications of our results for the hierarchy of subregular functions.

\subsection{Relation to Metrical Grid Theory}

The use of tiers and tier projection functions to investigate phonological complexity is of course not novel. One way of analyzing stress is in terms of so-called metrical grids (Liberman, 1975; Hayes, 1995; Kager, 1995; and many others), which are effectively tiers stacked on top of each other. The tiers of metrical grids correspond closely with the tiers used in this paper. In other words, our analysis aligns well with previous analyses of stress. Below we explain metrical grids, and highlight some similarities with the present paper.

The metrical grid below represents the word ælə 'bæmə 'Alabama' (Kager, 1995, p. 369). Tier 2 identifies the primary stress, tier 1 indicates all stresses, whether primary or secondary, and tier 0 shows the division of the word into syllables.

$\begin{array}{ccccc}\text { Tier 2 } & & & * & \\ \text { Tier 1 } & * & & * & \\ \text { Tier 0 } & * & * & * & * \\ & æ & \text { lə } & \text { bæ } & \text { mə }\end{array}$

Figure 1: The metrical grid for ,ælə'bæmə 'Alabama.'

Metrical grids can be used to understand the stress systems of Abkhaz and Lhasa Tibetan. The brief overview below follows Kager (1995), which interested readers should consult for a detailed analysis of both DO and DS systems. For Abkhaz, we begin with a tier 0 where $\mathrm{D}$ syllables have two asterisks, and $\mathrm{R}$ syllables one. In addition to the final syllable, any $* *$ on tier 0 not immediately followed by another $* *$ projects onto tier 1 . Finally, the leftmost tier 1 asterisk projects onto tier 2 . This gives grids like the one in Figure 2, for the word a-' $\underline{b}^{\mathrm{w} a k}$ 'jaməsa 'the poniard.'

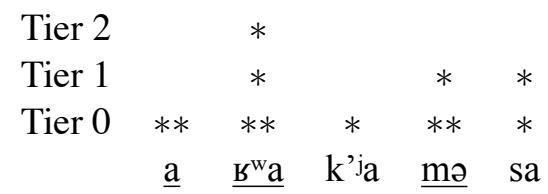

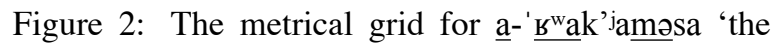
poniard.'

As we saw for English, a tier 2 asterisk identifies the primary stress. It is not clear whether tier 1 asterisks encode secondary stress in Abkhaz, but there are segmental alternations between [ə] and $[\varnothing]$ that are affected by tier 1 (see Spruit, 1986, pp. 73-77).

For words with no dominant syllables, such as maa-' k' ' 'one handle,' we simply project the rightmost syllable from tier 0 onto tier 1 . Again, the leftmost (and only) tier 1 asterisk projects onto tier 2.

$\begin{array}{cccc}\text { Tier 2 } & & & * \\ \text { Tier 1 } & & & * \\ \text { Tier 0 } & * & * & * \\ & \text { ma } & \text { a } & \text { k’o }\end{array}$

Figure 3: The metrical grid for maa-'k’ə 'one handle.'

For Lhasa Tibetan, we have no underlying accentual specifications, but instead place either one or two asterisks on tier 0 depending on whether the vowel is short or long. The leftmost tier $0 * *$ is projected onto tier 1.5 The leftmost $*$ of tier 1 projects onto tier 2 , indicating primary stress. For 'tý:tû́: 'shirt,' this gives the following.

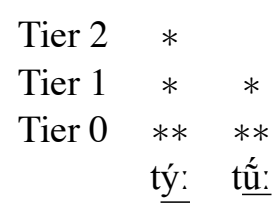

Figure 4: The metrical grid for 'tý́:tû́: 'shirt.'

\footnotetext{
${ }^{5} \mathrm{We}$ are not aware of data on secondary stress.
} 
In words without any long vowels, the same system produces the desired result. The word in Figure 5 is 'wòmá 'milk.' By convention, since tier 1 is empty, we project the leftmost $*$ of tier 0 instead (Kager, 1995, pp. 384-385).

$\begin{array}{ccc}\text { Tier } 2 & * & \\ \text { Tier } 1 & & \\ \text { Tier } 0 & * & * \\ & \text { wò } & \text { má }\end{array}$

Figure 5: The metrical grid for 'wòmá 'milk.'

In the analysis of Abkhaz, the projection of tier 1 mirrors the tier projection used in Proposition 7: both the asterisks and the projected Ds and Rs mark the location of Ds not followed by Rs, and both projections are ISL. The projection of tier 2 is ISL if tier 1 is taken to be the input, in the same way that the Abkhaz stress function reflects a strictly local dependency enforced over an ISL tier. In the analysis of Tibetan, the decomposition of $\tau$ into $g$ and $h$ is analogous to the convention that tier 2 is projected from tier 0 if tier 1 is empty.

\subsection{The Subregular Hierarchy}

Our work makes two contributions to the study of the subregular hierarchy. Firstly, our definition of the I-TISL functions naturally incorporates the notion of tier projection developed by Graf and Mayer (2018) into the family of strictly local functions proposed by Chandlee (2014), Chandlee et al. (2015), and Chandlee et al. (In prep). Secondly, we have presented an argument based on stress assignment that McCollum et al.'s (2018) definition of weak determinism is more natural for computational phonology than that of Heinz and Lai (2013).

Intuitively, the difference between DO and DS stress systems is that the former has a consistent directionality, while the latter does not. In our implementation of the Akbhaz stress function, the input is scanned from left to right, and when no appropriate D syllable is found, default final stress is assigned at the end of the computation. Thus, the Abkhaz stress function may be viewed as a "leftto-right" process. The contribution of weak determinism to the Tibetan stress function is that the right-subsequential component allows the process to "change direction" when no $\mathrm{H}$ syllable is found. If bidirectionality is the primary contribution of weak determinism to subregular phonology, then it may be desirable to impose additional structure on the two components of a weakly deterministic function. In Proposition 15, for example, $g$ is 2TISL, while $h$ is right 1,1 -IOSL.

\section{Conclusion}

This paper has considered unbounded stress systems in relation to the subregular hierarchy for functions. We have shown that the functions for assigning default-to-opposite (DO) and default-tosame (DS) stress are not part of the same subregular classes. The DO stress function in Abkhaz is subsequential, and belongs to the input tierbased input strictly local (I-TISL) class, which also captures other DO stress systems. The tiers and tier projection functions that we use are linguistically interpretable, sharing many properties with the phonological representations used in metrical analyses of stress. However, we have seen that the DS stress function in Lhasa Tibetan is not subsequential. DS stress can instead be captured using the class of weakly deterministic functions, and therefore we favor McCollum et al.'s (2018) definition of weak determinism over that of Heinz and Lai (2013).

\section{References}

Hyunah Baek. 2018. Computational representation of unbounded stress: Tiers with structural features. In Proceedings of CLS 53 (2017), volume 53, pages 1324, Chicago, IL. Chicago Linguistic Society.

Jane Chandlee. 2014. Strictly Local Phonological Processes. PhD Dissertation, University of Delaware, Newark, DE.

Jane Chandlee, Rémi Eyraud, and Jeffrey Heinz. 2015. Output Strictly Local Functions. In Proceedings of the 14th Meeting on the Mathematics of Language, pages 112-125, Chicago, IL. Association for Computational Linguistics.

Jane Chandlee, Rémi Eyraud, and Jeffrey Heinz. In prep. Input-output strictly local functions and their efficient learnability.

Willa Dawson. 1980. Tibetan Phonology. PhD Dissertation, University of Washington, Seattle, WA.

Vladimir Antonovič Dybo. 1977. Западнокавказкая аксентная система и проблема ее происхождения. In Конференция «Ностратические языки и ностратическое языкознание»: Тезисы докладов, pages 41-45, Moscow, Soviet Union. USSR Academy of Sciences. 
Calvin C. Elgot and Jorge E. Mezei. 1965. On Relations Defined by Generalized Finite Automata. IBM Journal of Research and Development, 9(1):47-68.

Matthew Gordon. 2007. Syllable Weight: Phonetics, Phonology, Typology. Routledge, London, United Kingdom.

Thomas Graf and Connor Mayer. 2018. Sanskrit nRetroflexion is Input-Output Tier-Based Strictly Local. In Proceedings of the Fifteenth Workshop on Computational Research in Phonetics, Phonology, and Morphology, pages 151-160, Brussels, Belgium. Association for Computational Linguistics.

Bruce Hayes. 1995. Metrical Stress Theory: Principles and Case Studies. University of Chicago Press, Chicago, IL.

Jeffrey Heinz and Regine Lai. 2013. Vowel Harmony and Subsequentiality. In Proceedings of the 13th Meeting on the Mathematics of Language (MoL 13), pages 52-63, Sofia, Bulgaria. Association for Computational Linguistics.

Jeffrey Heinz, Chetan Rawal, and Herbert G. Tanner. 2011. Tier-based Strictly Local Constraints for Phonology. In Proceedings of the 49th Annual Meeting of the Association for Computational Linguistics: Human Language Technologies, pages 58-64, Portland, OR. Association for Computational Linguistics.

René Kager. 1995. The Metrical Theory of Word Stress. In John A. Goldsmith, editor, The Handbook of Phonological Theory, 1 edition, Blackwell Handbooks in Linguistics, pages 367-402. WileyBlackwell, Oxford, United Kingdom.

Mark Yoffe Liberman. 1975. The Intonational System of English. PhD Dissertation, Massachusetts Institute of Technology, Cambridge, MA.

Connor Mayer and Travis Major. 2018. A Challenge for Tier-Based Strict Locality from Uyghur Backness Harmony. In Formal Grammar 2018, 23rd International Conference, FG 2018, Sofia, Bulgaria, August 11-12, 2018, Proceedings, volume 10950 of Lecture Notes in Computer Science, pages 62-83, Berlin, Germany. Springer Berlin Heidelberg.

Adam McCollum, Eric Baković, Anna Mai, and Eric Meinhardt. 2018. The expressivity of segmental phonology and the definition of weak determinism. LingBuzz, lingbuzz/004197.

David Odden. 1979. Principles of Stress Assignment: A Crosslinguistic View. Studies in the Linguistic Sciences, 9(1):157-176.

José Oncina, Pedro Garcia, and Enrique Vidal. 1993. Learning Subsequential Transducers for Pattern Recognition Interpretation Tasks. IEEE Transactions on Pattern Analysis and Machine Intelligence, 15(5):448-458.
George N. Raney. 1958. Sequential Functions. Journal of the Association for Computing Machinery, 5(2):177-180.

James Rogers. 1997. Strict $\mathrm{LT}_{2}$ : Regular :: Local : Recognizable. In Logical Aspects of Computational Linguistics: First International Conference, LACL '96 Nancy, France, September 23-25, 1996 Selected Papers, volume 1328 of Lecture Notes in Computer Science, pages 366-385, Berlin, Germany. Springer Berlin Heidelberg.

Jacques Sakarovitch. 2009. Elements of Automata Theory. Cambridge University Press, Cambridge, United Kingdom.

Caitlin Smith and Charlie O'Hara. 2019. Formal Characterizations of True and False Sour Grapes. In Proceedings of the Society for Computation in Linguistics, volume 2, pages 338-341, Amherst, MA. ScholarWorks@UMass Amherst.

Arie Spruit. 1986. Abkhaz Studies. PhD Dissertation, Leiden University, Leiden, Netherlands.

Loren Trigo. 1992. Abkhaz Stress Shift. In Brian George Hewitt, editor, Caucasian Perspectives, pages 191-235. Lincom Europa, Munich, Germany. 